DR 1

Figure DR1-1
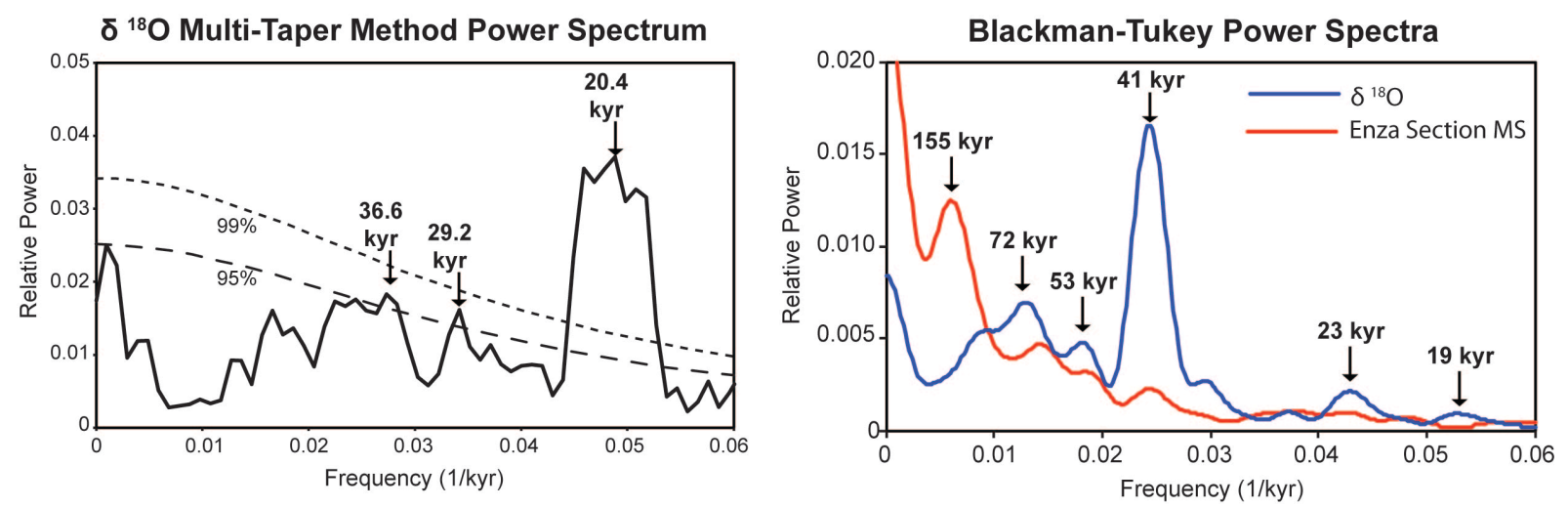

Figure DR1-1- We performed multi-taper method (MTM) (Thompson, 1982; 1990) and BTukey spectral analysis (reviewed in Weedon, 2003) on the global stacked $\delta^{18} \mathrm{O}$ curve from Lisiecki and Raymo (2005) between 0.7-1.7 Ma to see if significant spectral peaks occur at the same frequencies as those in the Enza section $\chi$ data series. The power spectrum on the left is the result of our MTM analysis and shows significant peaks aligned with obliquity and precession frequencies. Of particular note is the wide, slightly misaligned obliquity peak at $1 / 36.6 \mathrm{kyr}$, which is very similar to the $1 / 35.7 \mathrm{kyr}$ peak observed in the Enza $\chi$ MTM power spectrum (Figure 7, main text). For comparison, the figure on the right shows the results of our B-Tukey spectral analysis for both the stacked $\delta^{18} \mathrm{O}$ record and the Enza $\chi$ data series. Both records show peaks at $1 / 41 \mathrm{kyr}$, though the $\delta^{18} \mathrm{O}$ peak has more power. Both spectra have peaks at $\sim 1 / 72 \mathrm{kyr}$ and 1/55 kyr, similar to the spectral peaks observed in the Enza $\chi$ MTM power spectrum. These cycles are probably associated with transient frequencies that are known to occur during the middle Pleistocene (Hinnov, 2000). 
Figure DR1-2
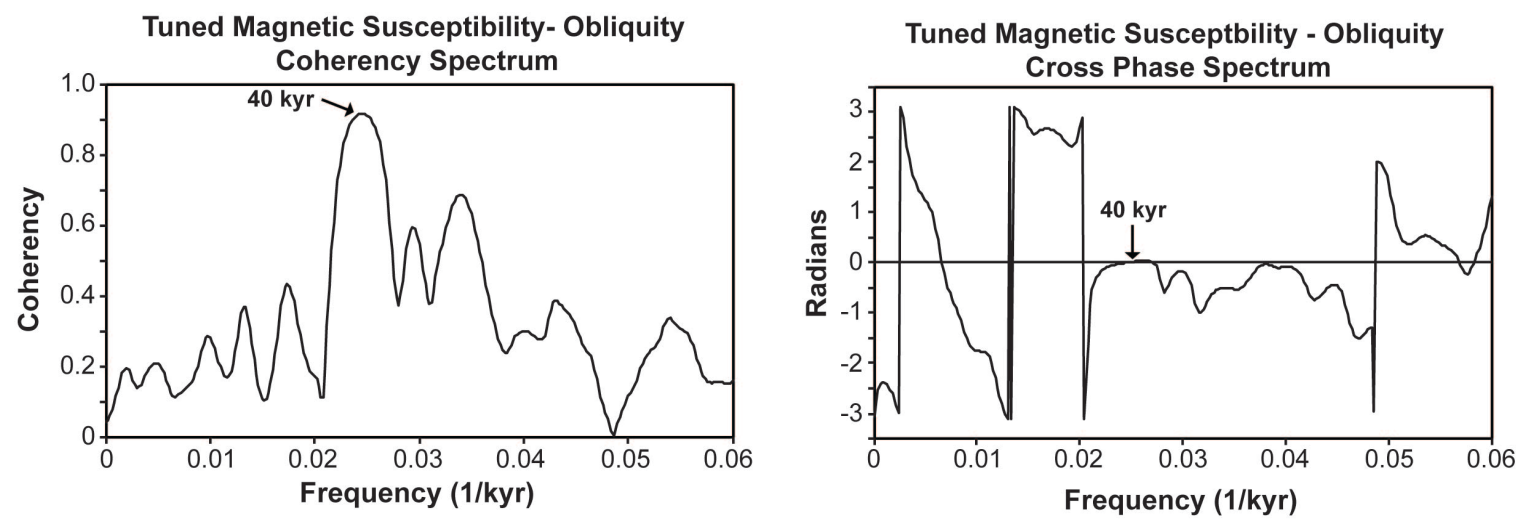

Figure DR1-2- To check the accuracy of our correlation of the 35 kyr cycle observed in the $\chi$ data series to Laskar et al.'s (2004) theoretical obliquity model, we calculated coherency (left) and cross phase (right) spectra between the tuned $\chi$ data series and the theoretical obliquity model. As expected, the two data series show coherency in the obliquity band (left). Additionally, the zero cross phase at the expected obliquity frequency (right) shows that the correlation shown in Figure 8 of the main text is correct and that we did not miss any obliquity cycles.

\section{Figure DR1-3}
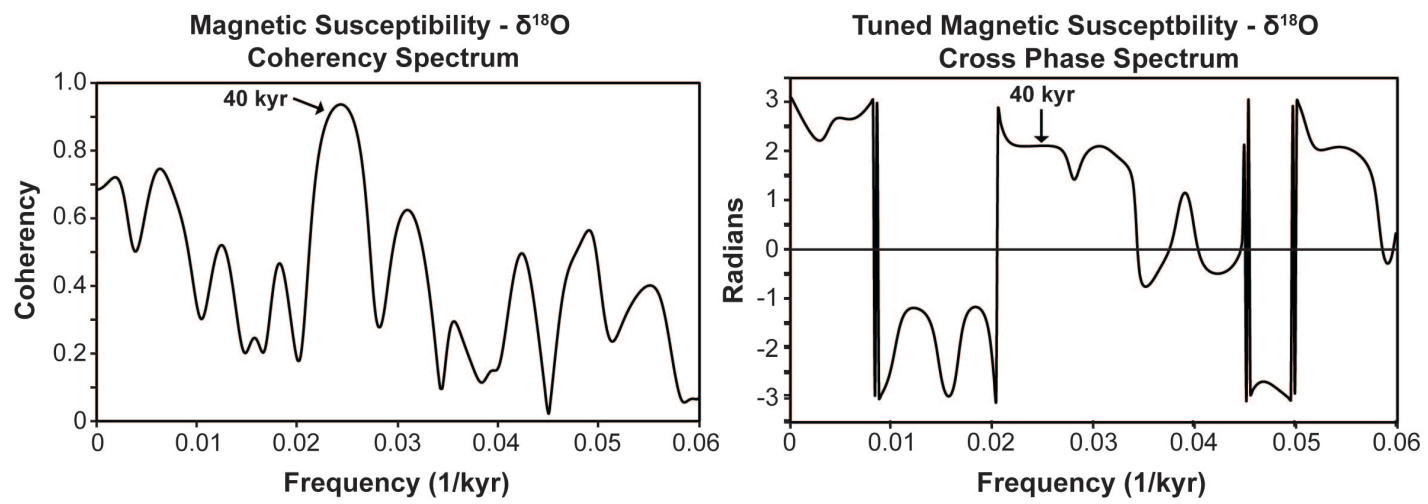

Figure DR1-3- Coherency (left) and cross phase (right) spectra between the tuned $\chi$ data series and the global stacked $\delta^{18} \mathrm{O}$ curve from Lisiecki and Raymo (2005) between 0.7-1.7 Ma. The two data series show coherency in the obliquity band, but a non-zero cross phase at the same 
frequency. This non-zero cross phase is expected since Lisiecki and Raymo (2005) tuned their $\delta^{18} \mathrm{O}$ to a lagged obliquity. This analysis provides additional evidence regarding the identification of Milankovitch cycles in the Enza section's $\chi$ data series and the efficacy of our correlation to the theoretical orbital model.

\section{REFERENCES}

Lisiecki, L.E., and Raymo, M.E., 2005, A Pliocene-Pleistocene stack of 57 globally disturbed benthic delta (super 18) O records: Paleoceanography, v. 20, p. 17, doi: 10.1029/2004PA001071.

Hinnov, L.A., 2000, New perspectives on orbitally forced stratigraphy: Annual Review of Earth and Planetary Sciences, v. 28, p. 419-475.

Thompson, D.J., 1990, Time series analysis of Holocene climate data: Philosophical Transactions of the Royal Society of London, Series A, Mathematical and Physical Sciences, v. 330, p. 601-616.

Thompson, D.J., 1982, Spectrum Estimation and Harmonic Analysis: Proceedings of the IEEE, v. 70, p. 1055-1096.

Weedon, G., 2003, Time-series analysis and cyclostratigraphy; examining stratigraphic records of environmental cycles: Cambridge, United Kingdom (GBR), Cambridge University Press, Cambridge, p. 259. 
DR 2- OSL Age Data

\section{USU-888}
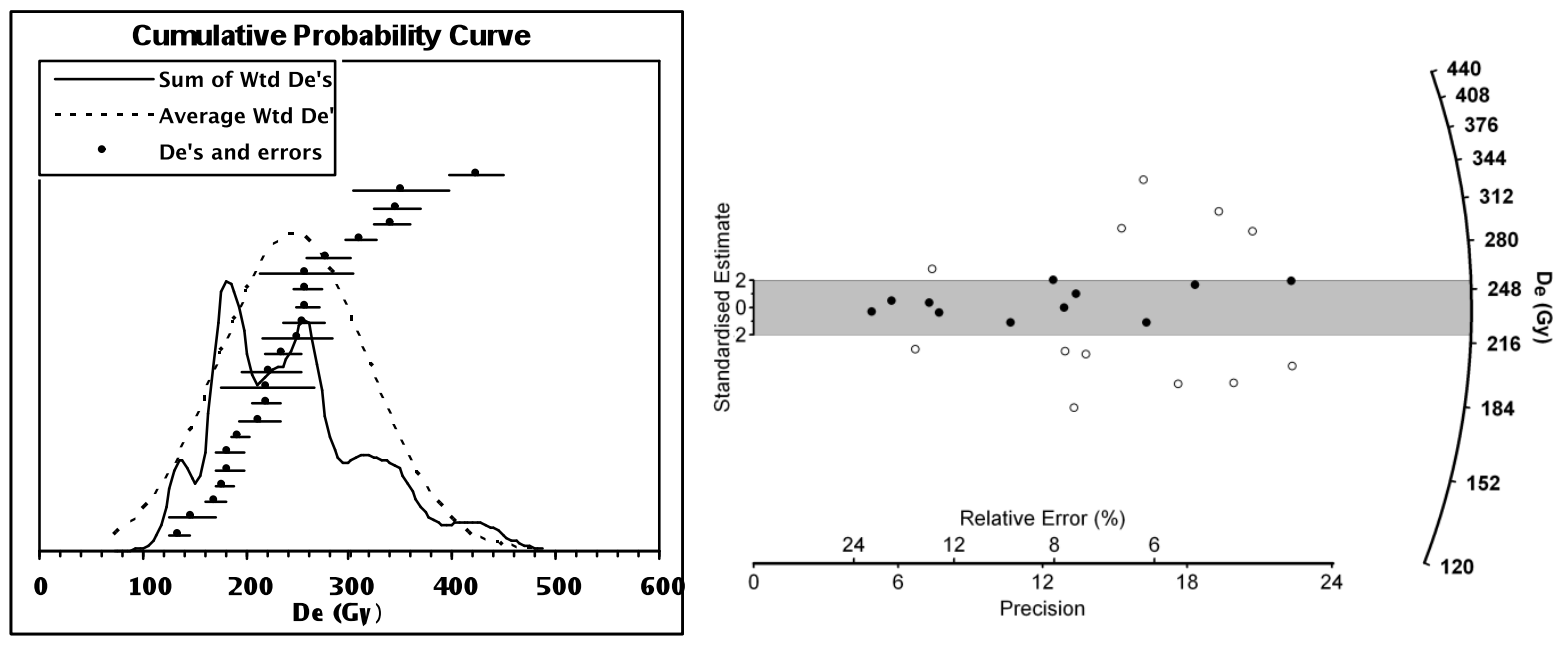

\section{USU-889}
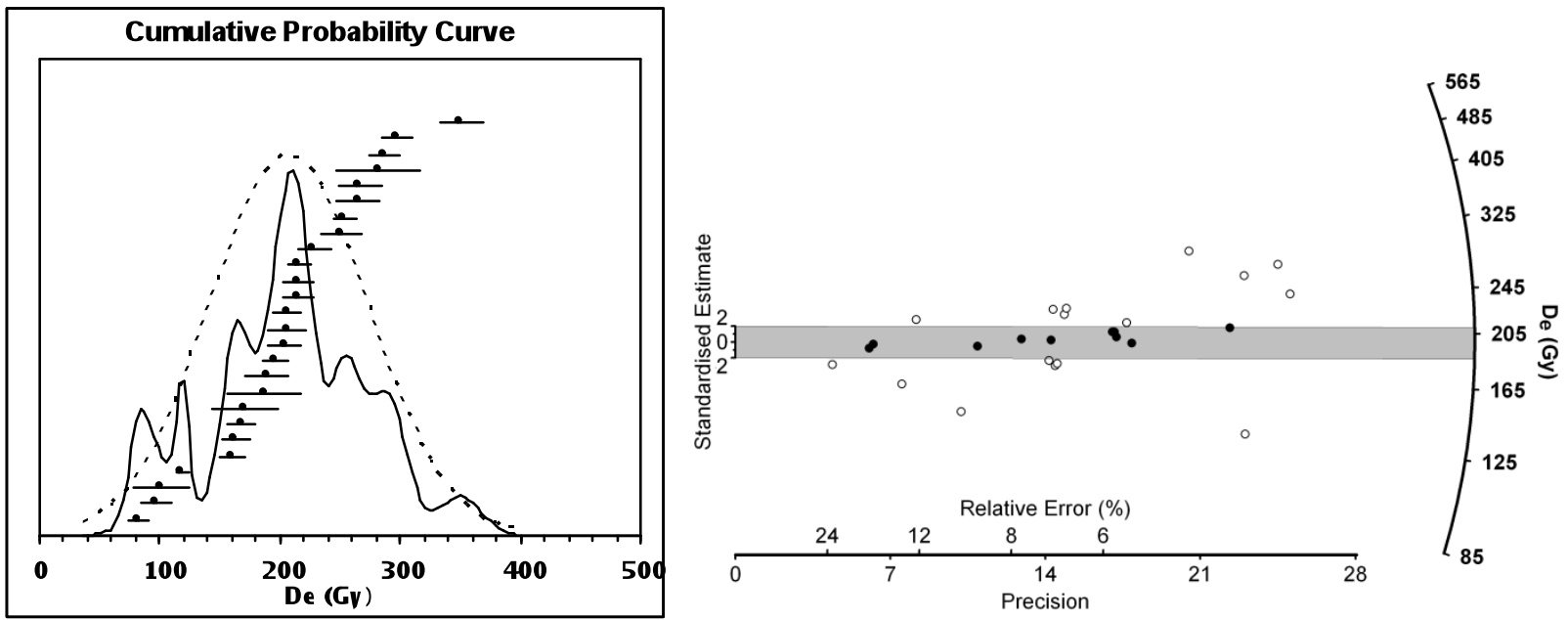

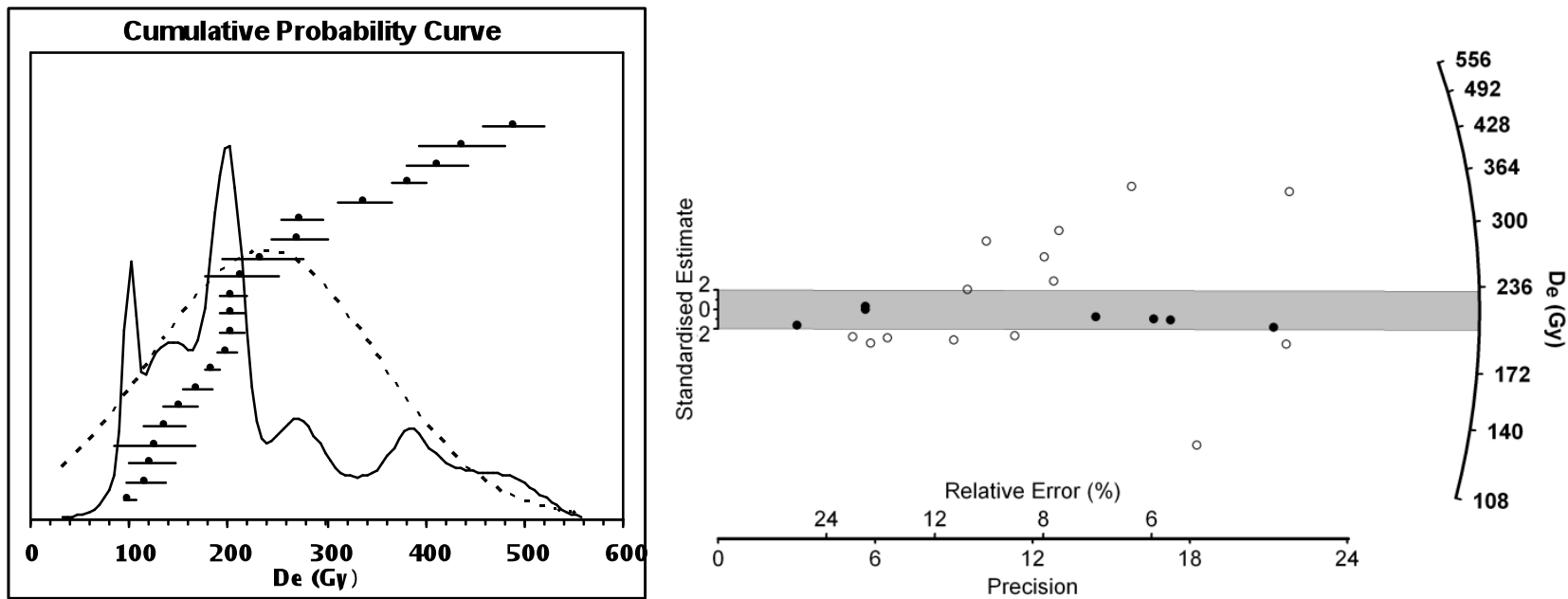

USU-891
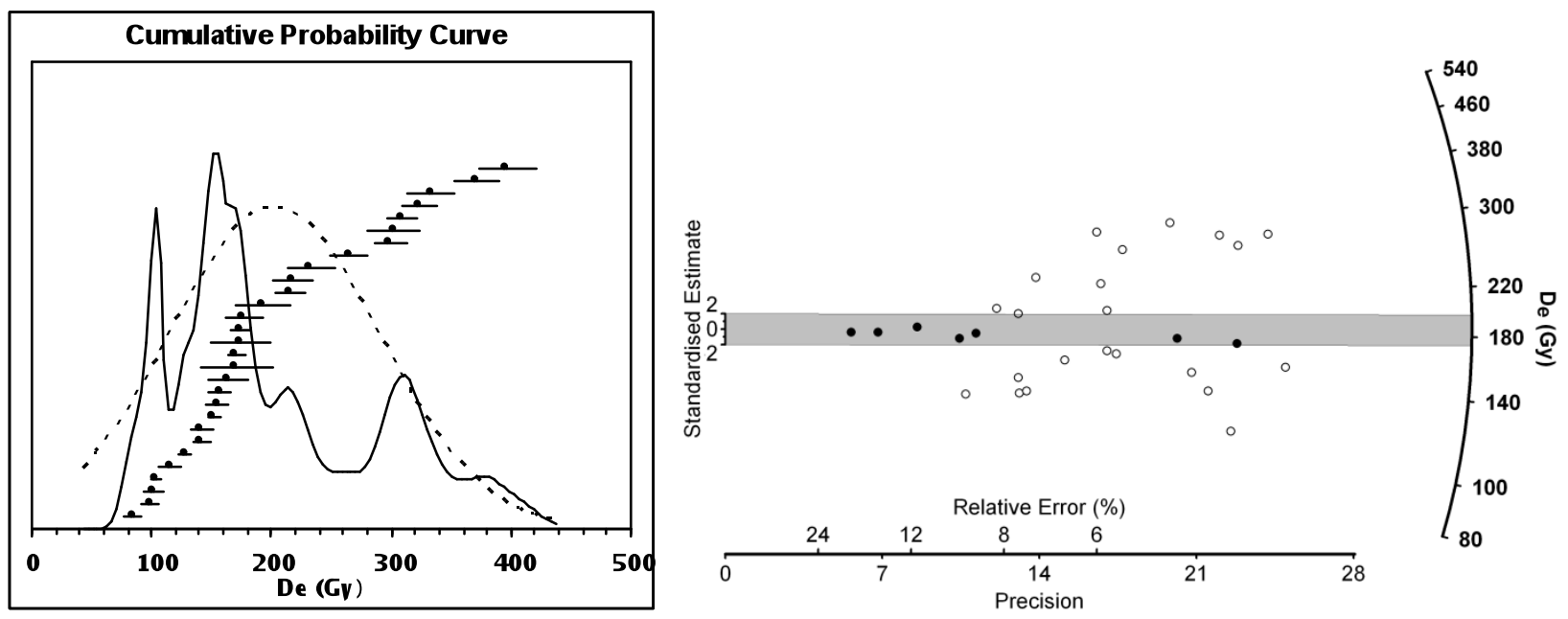

\section{USU-893}
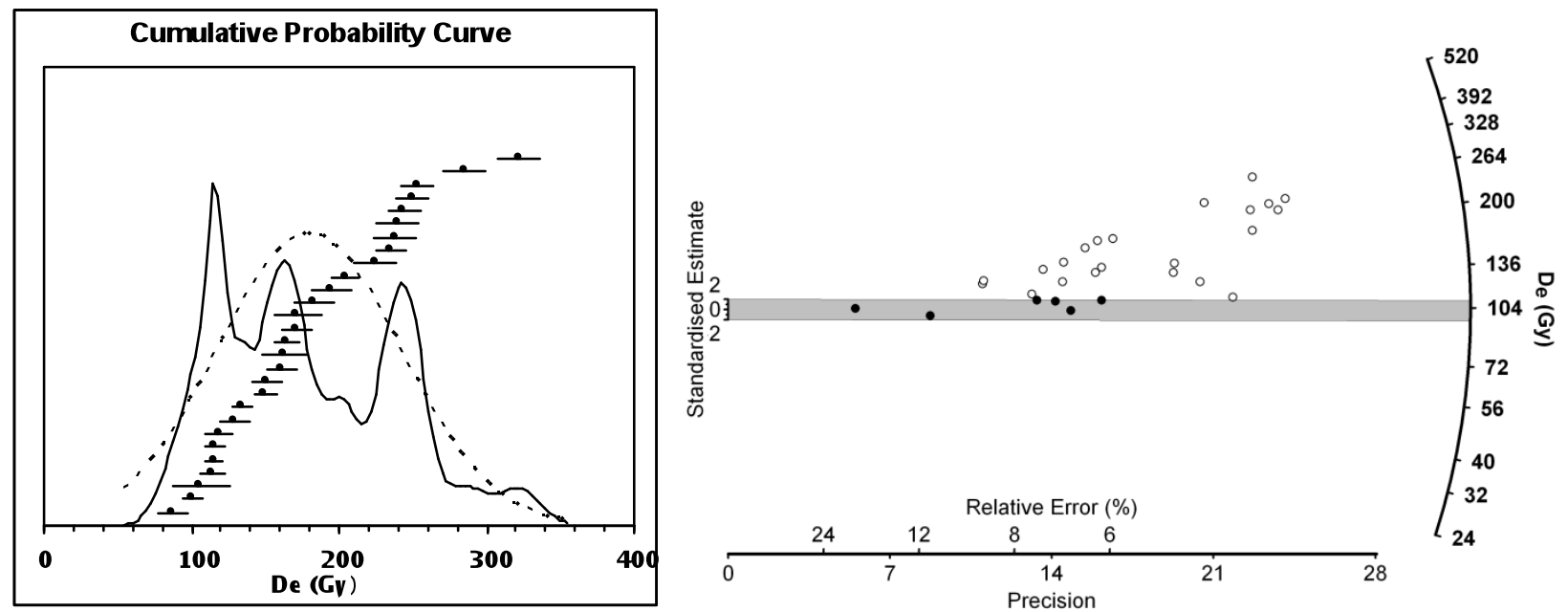

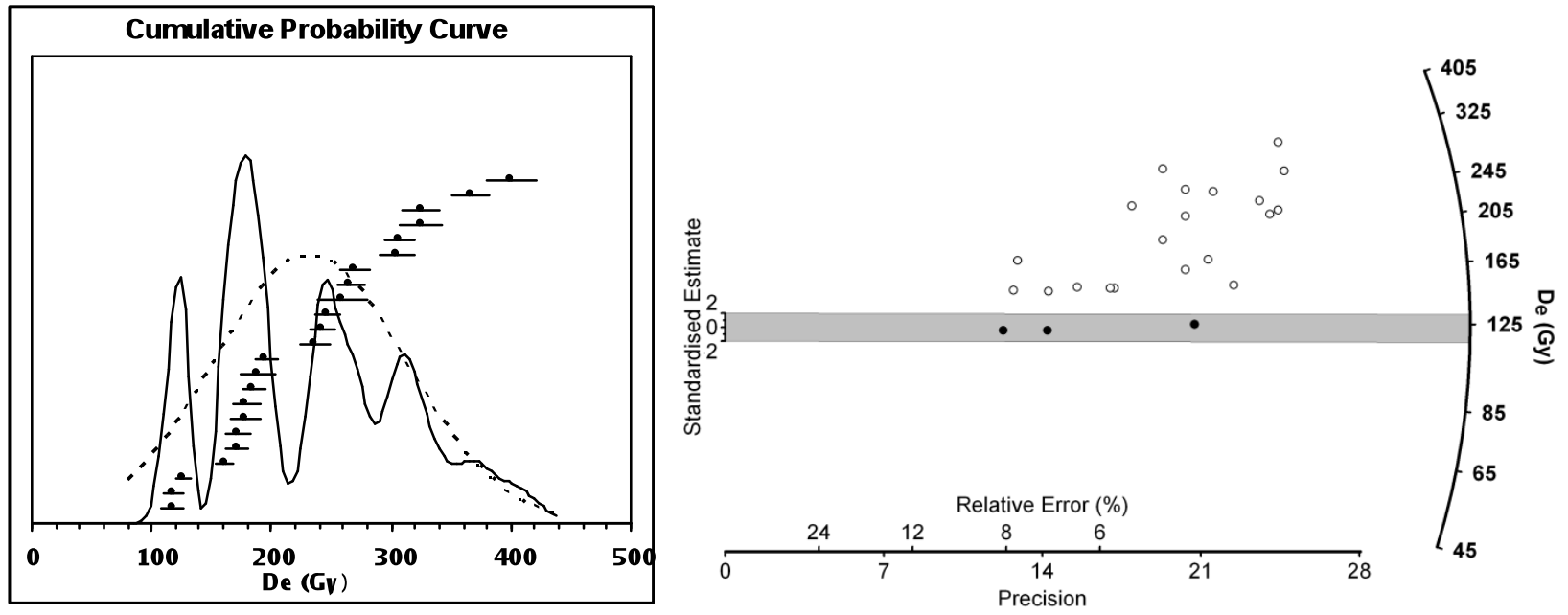

\section{USU-896}
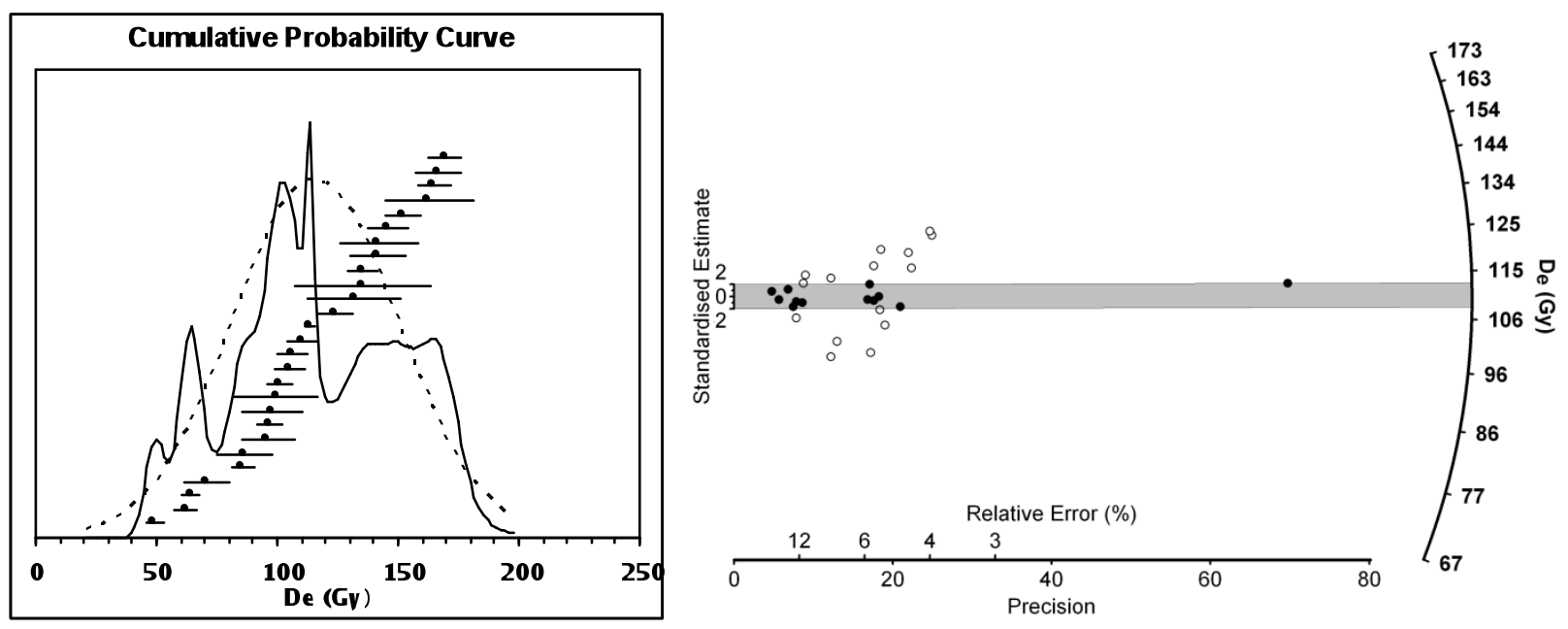

Figure DR-2 Cumulative probability distributions (solid black lines) of equivalent dose (De) for all samples from the Enza section. The De values with uncertainty ranges for individual aliquots are also plotted. The dashed lines represent a single peak average De distribution used to calculate the average burial age and uncertainties. For most of the samples the average De distribution represents a good fit to the cumulative probability distribution shown in the solid black line. The samples from $272 \mathrm{~m}$ (USU 890, USU 891), however, have a bi-modal distribution that is not well approximated by a single average $D e$ distribution. This is probably due to partial bleaching of these sediments. 
Therefore, for this sample we applied the minimum age model (MAM) (Galbraith et al., 1999) when calculating the sample burial age (see text for details).

\section{REFERENCES}

Galbraith, R.F., Roberts, R.G., Laslett, G.M., Yoshida, H., and Oleey, J.M., 1999, Optical dating of single and multiple grains of quartz from Jinmium Rock Shelter, northern Australia: Part I, experimental design and statistical models Archaeometry, v. 41, p. 339-364. 